\title{
Lateral approach of the dog brachial plexus for ventral root reimplantation
}

\author{
P Moissonnier $^{1,2}$, Y Duchossoy ${ }^{2,3}, \mathrm{~S}_{\text {Lavieille }}{ }^{1}$ and $\mathrm{J}-\mathrm{C}$ Horvat $^{3}$ \\ ${ }^{1}$ Service de Chirurgie; ${ }^{2}$ Laboratoire de Neurobiologie d'Alfort Ecole Nationale Vétérinaire d'Alfort, 7, Avenue du \\ Général De Gaulle, 94700 Maisons Alfort, France; ${ }^{3}$ Laboratoire de Neurobiologie, URA CNRS 1448, UFR \\ Biomédicale des Saints Pères, Université René Descartes, 45 rue des Saints Pères, 75270 Paris Cedex 06, France
}

\begin{abstract}
A lateral surgical approach of the cervical spinal cord and brachial plexus was developed in nine dogs for avulsion and reimplantation of the ventral cervical spinal roots (C). The surgical steps involved in exposing the spinal cord and roots are described. The avulsed rootlets of C6 and $\mathrm{C} 7$ were reimplanted in their initial position. As a direct consequence of the avulsion, flaccid paralysis of the shoulder and severe amyotrophy developed within 5-7 weeks on the injured side. In addition, the dogs exhibited clinical signs resulting from damage to long fiber tracts due to the reimplantation procedure. A partial recovery of these deficits was observed during the 6 postoperative months. Retrograde axonal tracing with horseradish peroxidase applied to the distal stump of the musculocutaneous, suprascapular, and subscapular nerves (originating from $\mathrm{C} 5, \mathrm{C} 6$ and $\mathrm{C} 7$ ) revealed the presence of labelled neuronal somata that were located in the ipsilateral ventral horn, close to the tip of the reimplanted rootlets. It is concluded that the dog constitutes a worthwhile animal model for the study of avulsion and reimplantation of brachial plexus root via a lateral surgical approach.
\end{abstract}

Keywords: avulsion; axonal regrowth; brachial plexus; dog reimplantation; ventral root

\section{Introduction}

Nerve root avulsions, commonly observed in most brachial plexus injuries, result in permanent paralysis of the muscles innervated by the avulsed roots and in sensory loss in the corresponding dermatomes. Spontaneous regeneration is not observed, not only because of the importance of the size of the gap separating the spinal cord from the avulsed rootlets, but also because of a fundamental lack of axonal regrowth and extension from neurons injured in the central nervous system (CNS). However, this inability for axonal regeneration cannot be considered as an intrinsic property of CNS neurons as most of them can extend an axon again along an autologous peripheral nerve segment grafted in their immediate vicinity. $1,2,3$ Root reimplantation, a new surgical strategy for the treatment of brachial plexus injuries, is based on these experimental data.

The first attempt to repair ventral avulsed roots was carried out via a dorsal approach in dogs. ${ }^{4}$ One avulsed ventral root was reimplanted ventrolaterally in the spinal cord. The dorsal approach was successfully used in the rat $^{5}$ and in the sheep ${ }^{6}$ cervical spinal cord, or in the cat lumbosacral plexus. ${ }^{7}$ However, the reimplantation of the ventral root into its precise spinal site of origin cannot be accomplished via a dorsal approach. A ventral surgical approach was

Correspondence: P Moissonnier developed also in the cat. ${ }^{8}$ A single ventral root of the plexus was reached and a high mortality rate was observed because of blood loss initiated by the surgical procedure. In the monkey, ${ }^{9,10}$ after all the ventral roots of the brachial plexus were avulsed and replaced ventrolaterally via a lateral approach, motor recovery was observed.

Yet, a number of problems still remain to be solved before a routine clinical application of reimplantation can be performed. After avulsion, roots retract and are sometimes found, far away from the cord, in the ventral cervical region which cannot be explored via a dorsal approach. It is also difficult to tract them into the vertebral canal for the reimplantation procedure. Traumatic avulsions of the brachial plexus roots, the most common neurological condition in the dog forelimb, appear to be very similar in dog and in man. ${ }^{11}$ In this way, the dog is a good clinical model. In a previous report we have demonstrated the ability of lumbar neurons of the adult dog to extend at least 10 to $15 \mathrm{~cm}$ long axons into peripheral nerve segments grafted close to their neuronal somata. ${ }^{12}$ These positive results of axonal regrowth direct toward application on roots reimplantation in the dog.

Thus, the aims of our study are: (1) to investigate, in a dog model, a lateral surgical approach for the exploration of the cranial part of the brachial plexus and reimplantation of, at least, three ventral roots in the precise area where the avulsion had taken place; 
(2) to evaluate whether axonal regeneration, after root avulsion and reimplantation, does occur in the dog; (3) to describe the consequences of the surgical approach, avulsion and reimplantation on dog gait.

\section{Materials and methods}

Dogs handling and surgical procedures were reviewed and approved by the Scientific Board of the Veterinary School of Maisons Alfort, France. The surgical approach was initially developed by dissection studies in fresh dog cadavers.

\section{Animals}

Nine healthy adult male ${ }^{1}$ and female ${ }^{8}$ beagle dogs (body weight $13-15 \mathrm{~kg}$ ) underwent root avulsion and reimplantation.

Prior to surgery, the dogs were given cephalexine (Rilexine, Reading, $30 \mathrm{mg} / \mathrm{kg}$ intravenously) and methylprednisolone sodium succinate (Solumedrol, Upjohn, $30 \mathrm{mg} / \mathrm{kg}$ intravenously). Ketoprofen (Ketofen $10 \%$, Rhône Mérieux, $2.2 \mathrm{mg} / \mathrm{kg}$ ) was administered intravenously for pain control. The skin over the left forelimb, shoulder and caudal cervical region was prepared for aseptic procedure. The general anaesthesia was induced with intravenous injection of diazepam (Valium, Roche, $0.2 \mathrm{mg} / \mathrm{kg} \mathrm{IV)} \mathrm{and} \mathrm{thio-}$ barbital (Nesdonal, Rhône Mérieux, $15 \mathrm{mg} / \mathrm{kg}$ IV), and maintained with oxygen $(100 \%)$ - isoflurane $(2 \%) \quad$ Forane $^{\mathbb{R}}$, Abbott) using a Bain inhalator. During the surgery, the dogs were perfused with a saline solution $(15 \mathrm{ml} / \mathrm{kg} / \mathrm{h})$. They were secured on the table in right lateral recumbancy.

\section{Surgical approach of the cervical column}

The transverse process of the sixth cervical vertebra was palpated.

\section{Skin incision}

A $12 \mathrm{~cm}$ curved incision was made $3-4 \mathrm{~cm}$ cranially from the midpoint of the cranial border of the scapula to a point located slightly distal to the greater tubercle of the humerus. The platysma and fascia cervicalis were incised exposing the cleidocervicalis, omotransversarius and trapezius muscles (Figure 1). The superficial cervical artery and vein, which emerge between the cleidocervicalis and the trapezius muscles, were ligatured. The superficial cervical lymph node, lying medially to the superficial cervical artery and vein, was retracted caudally.

\section{Desinsertion of the omo-transversarius muscle}

The omotransversarius muscle was incised near its insertion on the spine of the scapula and withdrawn cranially (Figure 2). The dissection was continued medially through the deep fascia along the dorsal

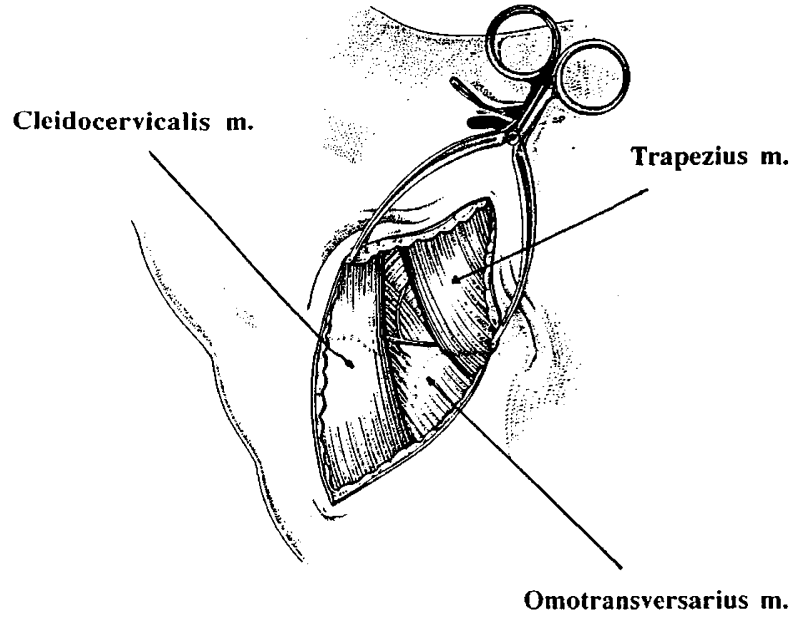

Figure 1 Surgical approach: superficial plane. The skin has been incised. The superficial musculature (trapezius, omotransversarius and cleidobrachialis muscles) is identified

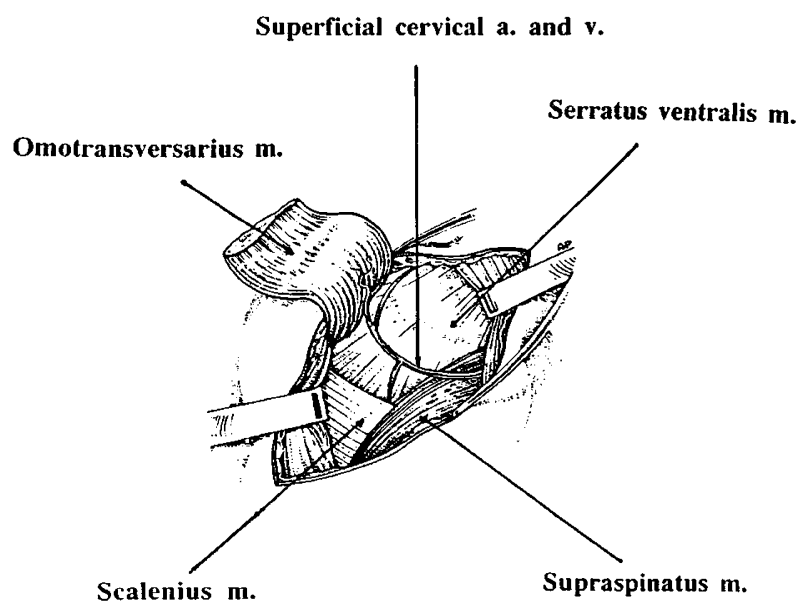

Figure 2 Surgical approach: Desinsertion of the omotransversarius muscle from the spinous process of the scapula

border of the cleidocervicalis muscle which was withdrawn ventrally. A self retaining Gelpi retractor was positioned between the cleidocervicalis and the trapezius muscles. The scapula was withdrawn caudally with a Farabeuf retractor. The extrathoracic part of the brachial plexus could be exposed and palpated ventrally.

Transection of the scalenius muscles

The ventral branches of the fifth (C5) to the eighth (C8) cervical and of the first thoracic (T1) nerves were exposed by transecting the superficial and deep portions of the scalenius muscle. The avulsed roots are generally found under the scalenius in the accidentally traumatized dogs. When the ventral 
branch of $\mathrm{C} 5$ was incorporated into the brachial plexus, we cut it to allow a complete denervation of the cranial part of the plexus. ${ }^{13,14}$ In particular, there was no contribution of the ventral branch of $\mathrm{C} 8$ to the suprascapular nerve in the dogs we used.

Elevation of the muscles from the dorsal surface of the transverse processes of the cervical vertebrae 5, 6 and 7 The longissimus cervicis, longissimus capitis and serratus ventralis muscles were elevated from the bone with a periosteal elevator after their insertions were coagulated with a bipolar-forceps, and they were withdrawn dorsally (Figure 3).

Elevation of the muscles from the laterodorsal surface of the articular processes of the cervical vertebrae 5, 6 and 7

Semi-spinalis capitis and multifidus cervicis muscles were elevated from the articular processes, using the procedure previously described (Figure 4). Transection of the dorsal branches of the spinal nerves was attempted at this time of the procedure to facilitate their dorsal withdrawal. Muscle elevation was carried out laterally on the articular and transverse processes by desinserting intertransversarii ventralis cervicis and eventually intertransversarii dorsalis muscles.

\section{Hemilaminectomy}

Self retaining Gelpi retractors were inserted to maintain the exposure of the ventral branches and cervical vertebrae 5, 6 and 7. A large part of the articular facets was removed with a bone rongeur. The remaining part as well as the cranial and caudal vertebral laminae were removed with a high-speed air

\section{Serratus ventralis $\mathbf{m}$.}

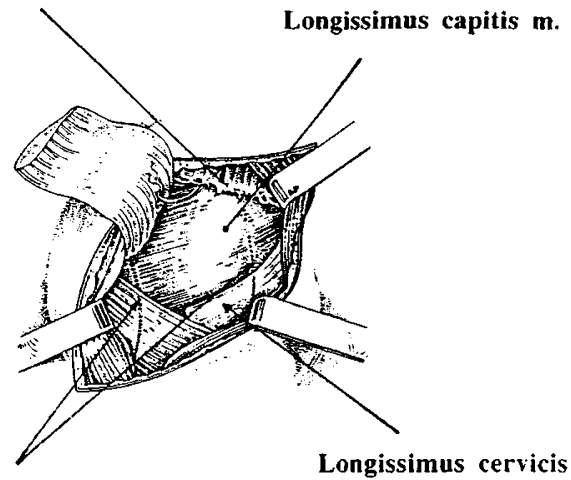

Ventral branches of $\mathrm{C} 6$ and $\mathrm{C} 7$

Figure 3 Surgical approach: Elevation of the muscles from the dorsal aspect of the transversarius processes. Longissimus cervicis, longissimus capitis and serratus ventralis muscles are retracted dorsally drill. Bleeding of the interspinous branches of the internal vertebral venous plexus, which penetrate the interarcuate ligament, was occasionally encountered and controlled with an haemostatic absorbable sponge (Surgicel, Johnson and Johnson). The hemilaminectomy (from C5-C7) was extended dorsally to the base of the spinous process and ventrally almost to the floor of the vertebral canal, stepping down to the internal vertebral venous plexus, which was preserved (Figure $5)$.

\section{Root avulsion and reimplantation}

The opening of the vertebral canal gave access to the dura which was firstly secured and then pulled laterally

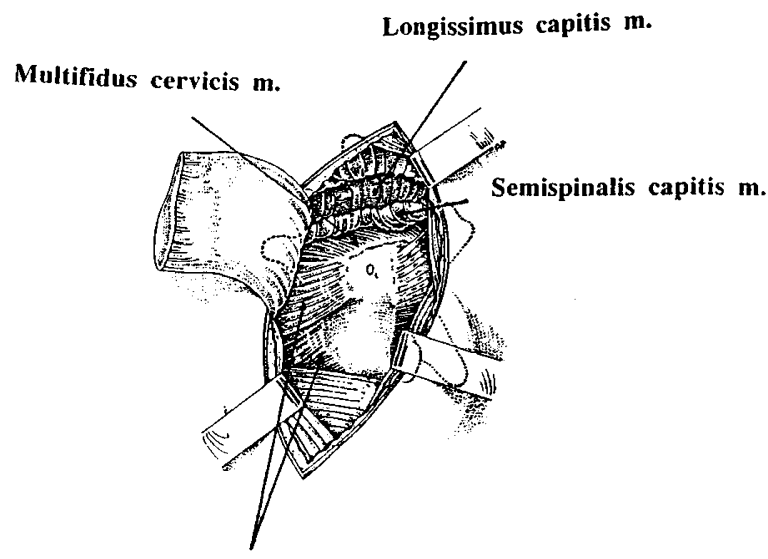

Intertransversarii ventralis and dorsalis $\mathbf{m m}$.

Figure 4 Surgical approach. Elevation of the muscles from the lateral and the dorsal aspects of the articular processes. The juxtavertebral musculature (semispinalis capitis, multifidus cervicis, intertransversarii ventralis and dorsalis $\mathrm{mm}$ ) has to be removed from the articular processes, to give access to the lateral aspect of the overlying vertebra

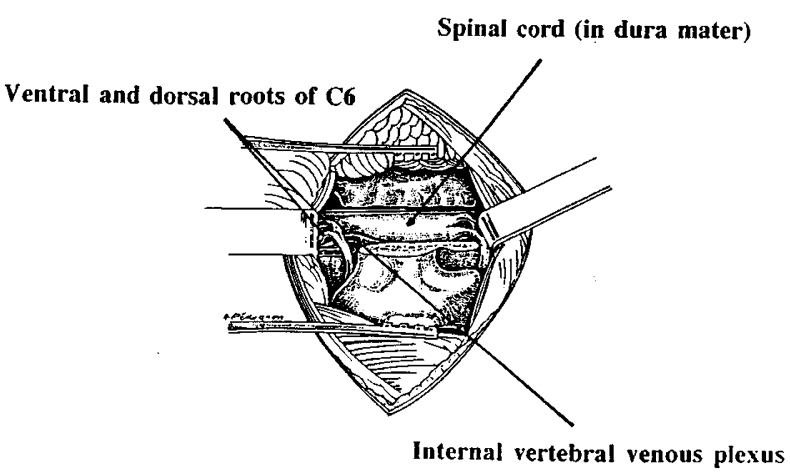

Figure 5 Surgical approach: Hemilaminectomy and facectomy. Facets and lateral lamina of the vertebra are resected, showing the spinal cord in its dura mater and, ventrally, the internal vertebral venous plexus 
with four stay sutures (4/0, Prolène, Ethicon). Under a dissecting microscope, the dura was incised longitudinally and dorsally to the ventral roots with a pair of microscissors. The spinal cord was gently rotated by placing two stay sutures into the denticulate ligament to improve the exposure of the sixth and seventh ventral cervical roots (C6 and $\mathrm{C} 7$ ). This rotation did not exceed $15-20^{\circ}$ in order to limit spinal cord injury. The rootlets were pulled in a caudal direction, parallel to their course, with a curved needle handler (Figure 6a). This procedure corresponds to a true avulsion in which axonal rupture is localized proximally to the central peripheral transition zone. A slit was made in the pia matter and spinal cord white matter, ventrally, with regard to the denticulate ligament, at the level of the lesion resulting from the avulsion of the rootlets. The $\mathrm{C} 6$ and $\mathrm{C} 7$ rootlets were reimplanted through the slit in their initial position into the grey matter (approximately $1-2 \mathrm{~mm}$ in depth) with a microforceps and covered with a small piece of haemostatic sponge (Surgicel, Johnson \& Johnson) (Figure 6b).
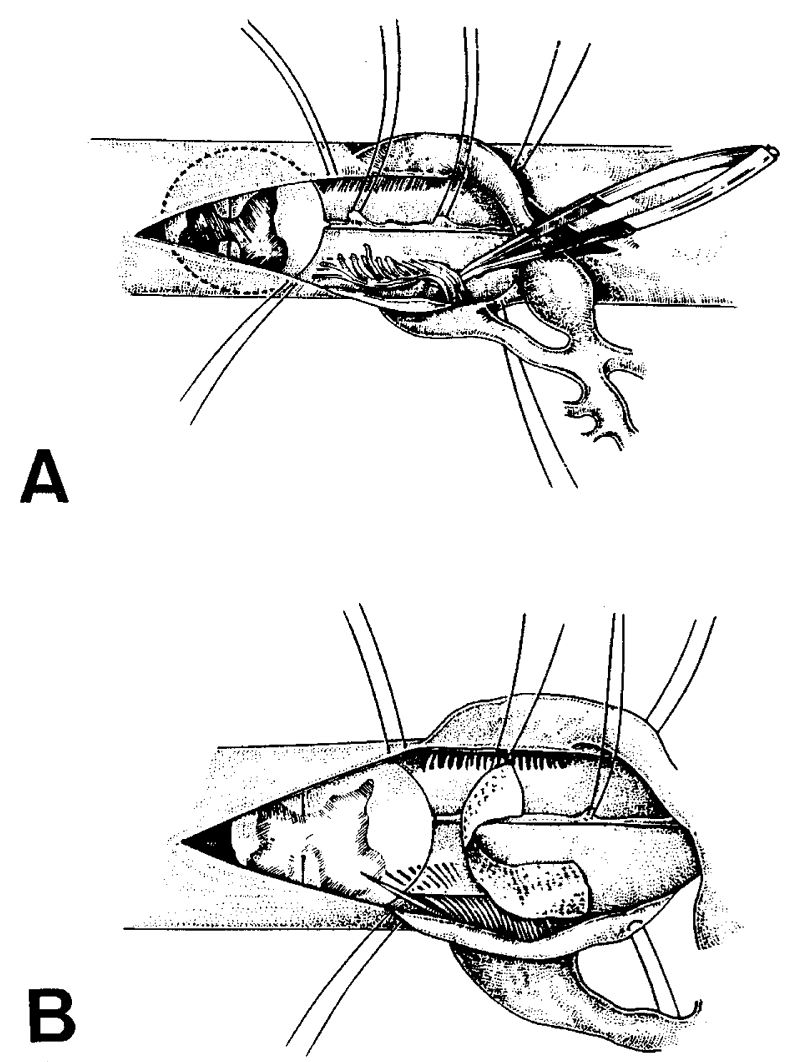

Figure 6 Semi-schematic view of the avulsion-reimplantation of one ventral root. The spinal cord is cross sectioned to show the depth of the ventral rootlet reimplantation. (a) The ventral root is avulsed from the spinal cord with a needle handler. (b) The avulsed rootlets are reimplanted into a slit in the spinal cord and covered with a small haemostatic sponge (Surgicel ${ }^{\mathbb{R}}$ )

\section{Closing procedure}

A fat autograft was placed on top of the hemilaminectomy site. The semi-spinalis capitis, multifidus cervicis, serratus ventralis, longissimus cervicis, longissimus capitis muscles were sutured together with the scalenius muscles (Vicryl, 3/10, Ethicon). The subcutaneous tissue and skin were closed in separate layers (Vicryl 2/10 and Ethicrin 2/10, Ethicon).

\section{Postoperative period}

Methyl prednisolone (Medrol, Upjohn) was administered orally $(1 \mathrm{mg} / \mathrm{kg})$ during 4 days. Behaviour, gait, and complete neurological function were assessed every day. We scored the neurological status with the following scales: Three parameters were considered for the left pelvic limb evaluation: motility, proprioception and patellar reflex. Motility was scored 2 (normal), 1 (monoparesia) or 0 (monoplegia). The patellar reflex was increased (0) or normal (1). Proprioception either disappeared (0), or decreased (1) or was normal (2). The left pelvic limb score corresponds to the sum of these three parameters. Thus, normal dogs were scored 5. In the same way, the ability to use the left thoracic limb (forelimb) was scored as 2 (normal), 1 (lameness with weight bearing), and 0 (monoparesia or lameness without weight bearing).

\section{Retrograde axonal tracing methods with Horse Radish} Peroxydase (HRP)

Six months after reimplantation, the dogs were anaesthetized again using the procedure described above and prepared for surgery. The craniomedial aspect of the left shoulder was approached, ${ }^{15}$ the musculocutaneous, suprascapular and subscapular nerves were exposed, cleared from surrounding tissues and transected. HRP (Sigma, type VI) was applied to the proximal stump of the nerves for $1 \mathrm{~h} .{ }^{16}$ The exposed area was then washed out with saline and the surgical wound was closed in layers.

\section{Euthanasia and spinal cord removal}

Forty-eight hours later, the dogs were sacrificed by barbiturate overdosing after deep sedation with ketamine. A medial sternotomy was created and the left ventricle of the heart was canulated. The dogs were at first perfused with an isotonic heparinized saline solution $(10-151)$, then fixed with $10-121$ of $3 \%$ glutaraldehyde in $0.1 \mathrm{M}$ phosphate buffer at $4{ }^{\circ} \mathrm{C}$. The cervical spinal cord was dissected out and kept in the same fixative. Each cervical spinal cord segment (5 to 8) was cut into $40 \mu \mathrm{m}$ thick cross section with a cryostat. The sections were processed for HRP histochemistry according to Mesulam ${ }^{16}$ and counterstained with neutral red. In every section, only labelled neurons displaying a nucleolus were counted with a light microscope. The distribution of the neurons in the spinal cord was plotted. 


\section{Results}

General behaviour

There was no postoperative complication and all the dogs were healthy before euthanasia.

\section{Neurological examination}

A flaccid paralysis of the left shoulder (no voluntary flexion and extension) appeared immediately after surgery in all the dogs. During the first 2 postoperative months, the dogs did not use their left thoracic limb which was held in extension along the thoracic wall (score 0). Within weeks a severe amyotrophy developed in the muscles supplied by the avulsed nerve roots (supraspinatus, infraspinatus and biceps brachialis muscles). These muscles were reduced to a thin strip of elastic tissue, contrasting with the bulky appearance of the muscle on the controlateral side. The first signs of clinical restoration were difficult to assess but a gradual improvement of function of the paralysed forelimb occurred and, 6 months after surgery, three dogs (numbers 7, 8 and 9) were able to use this forelimb (Figure 7).

All the dogs also exhibited neurological signs indicating damage to the long fiber tracts. Pelvic limb

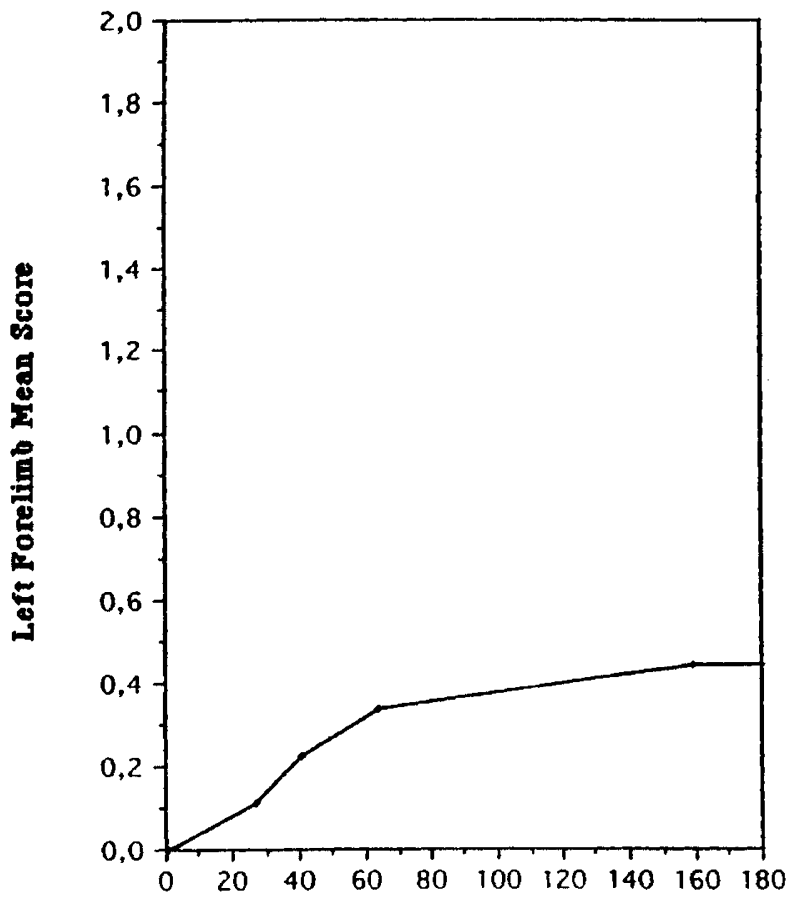

\section{Postoperative DAYS}

Figure 7 Clinical evaluation of left forelimb function. Function of the left forelimb was scored as 2 (normal), 1 (lameness with weight-bearing), and 0 (monoparesia or lameness with no weight-bearing) paraplegia or paresia, exaggerated spinal reflexes (patellar and flexion reflexes), proprioceptive deficits, and hypalgesia were observed. The spinal cord function progressively recovered (Figure 8) but the neurological score remained low even at the end of the study (mean score $=3.3$ ). The ocular sympathetic system was also injured by the reimplantation, resulting in a typical Claude Bernard-Horner's syndrome which disappeared before the fifteenth postoperative day (Figure 9).

\section{Histology}

The reimplanted roots were embedded in a scar tissue and their tips penetrated into the ventral horn of the grey matter.

Retrograde axonal tracing with HRP resulted in the labelling of 13-565 neuronal somata which were located close to the intraspinal tip of the rootlets scattered all over the ipsilateral ventral horn of spinal cord segments 6 and 7. Numerous primary sensory neurons also appeared to be labelled in the dorsal root ganglia close to the injury and grafting site. Figure 10 shows the labelled neuron distribution in C6 in dog number 7. With regard to their localization, size and morphology, most of these

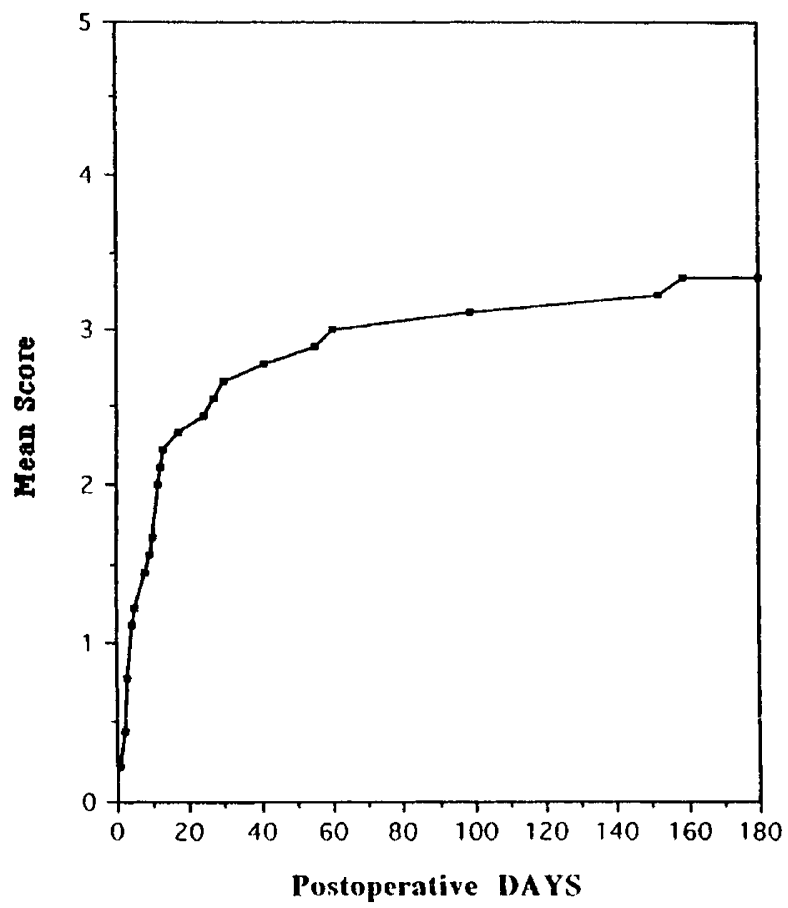

Figure 8 Clinical evaluation of the left hindlimb function. Hindlimb score is the sum of motility, reflex and proprioception scores. Motility is normal (2), diminished (1) or abolished (0), patellar reflex is increased $(0)$ or normal (1) and proprioception disappears (0), diminishes (1) or is normal (2). A normal dog is scored 5. 'Mean score' is the mean of the hindlimb scores of the nine dogs 


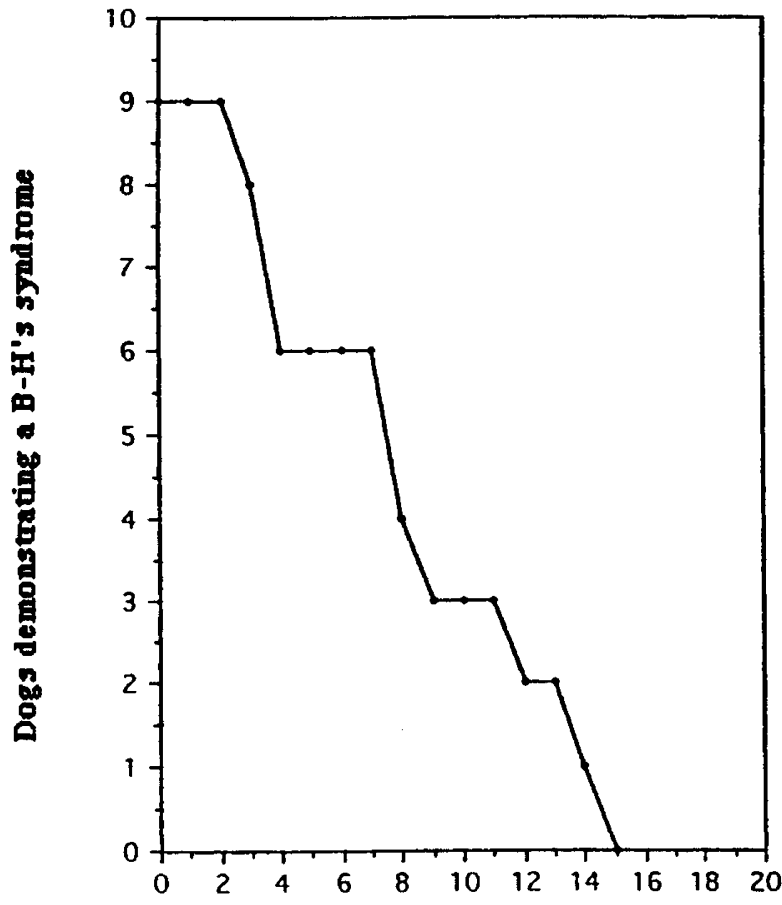

Postoperative DAYS

Figure 9 Evolution of the Claude Bernard-Horner's syndrome. Claude Bernard-Horner's syndrome (interruption or loss of sympathetic innervation) results in miosis, enophtalmos, protrusion of the third eyelid, ptosis of the upper eyelid and narrowing of the palperbral fissure

labelled cells were assumed to be motoneurons (Figure 11). No labelled neurons were identified in the contralateral $\mathrm{C} 5$ to $\mathrm{C} 8$ segments.

Cavitation of the spinal cord (micro and macro cyst formation) was a constant finding upon histological examination (Figure 11). These cysts were located in the grey matter near the intraspinal tips of the avulsed roots as well as at some distance from them (1$10 \mathrm{ml}$ ). They were surrounded by an acellular, amorphous lining membrane. The nerve cells located beside the cysts seemed to be unaffected. The bigger these cysts were, the fewer were the number of neurons labelled by HRP.

\section{Discussion}

The results reported in this study provide evidence that: (1) the dog appears to be a good model for the study of brachial plexus injury and repair; (2) our lateral surgical procedure allows a safe and reliable approach of the ventral roots of the brachial plexus and (3) extensive axonal regrowth from injured spinal neurons into reimplanted rootlets does occur.
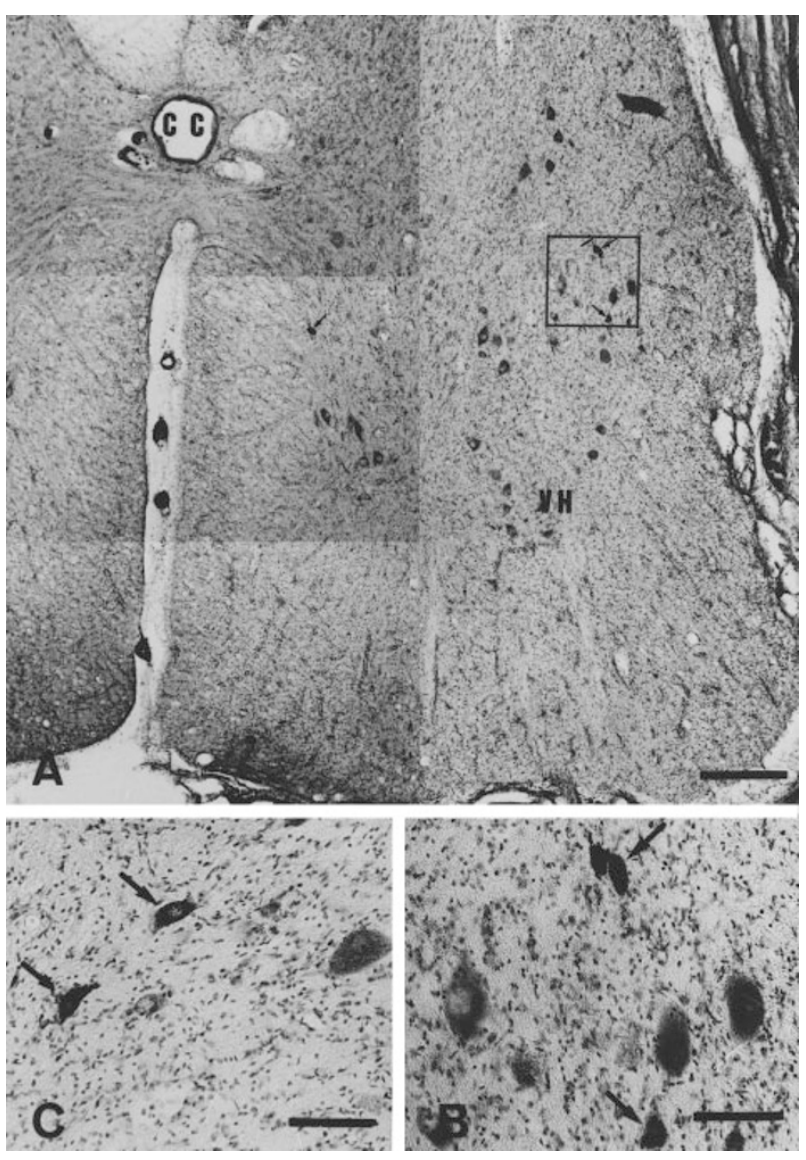

Figure 10 Retrograde neuronal labelling in the cervical spinal cord, 6 months after ventral root avulsion and reimplantation, HRP histochemistry using tetramethyl benzidine (TMB) and counterstaining with neutral red. (a) Cross section of the spinal cord at C7 level. Labelled neurons (small arrows) are those that have grown an axon through the reimplanted ventral root at least up to the site of tracer application (VH: Ventral Horn). (b) High magnification of labelled neurons (arrows) seen in a. (c) Labelled neurons (arrows) in another section. Scale bars: $A=400 \mu \mathrm{m}$; $\mathrm{B}=100 \mu \mathrm{m} ; \mathrm{C}=100 \mu \mathrm{m}$

The dog as an animal model for the study of brachial plexus injury and repair

Several animal models of root avulsion and reimplantation have been described. ${ }^{4-8,10}$ The selection of the most appropriate species for such studies is a major issue, as far as the animal model can constitute a useful reference for possible clinical application in mankind. Like a man, brachial plexus injuries are occasionally encountered in the veterinary practice, especially in the dog. ${ }^{11,17}$ In this animal, traction lesions of the brachial plexus are usually the result of accidents and consist both in spinal nerve root rupture, and avulsion of one or several roots from the spinal cord. As in man, spontaneous reconstruction has never been observed and surgical repair is still at present a difficult task.

The reestablishment of appropriate connections following surgery implies axonal regrowth from spinal 

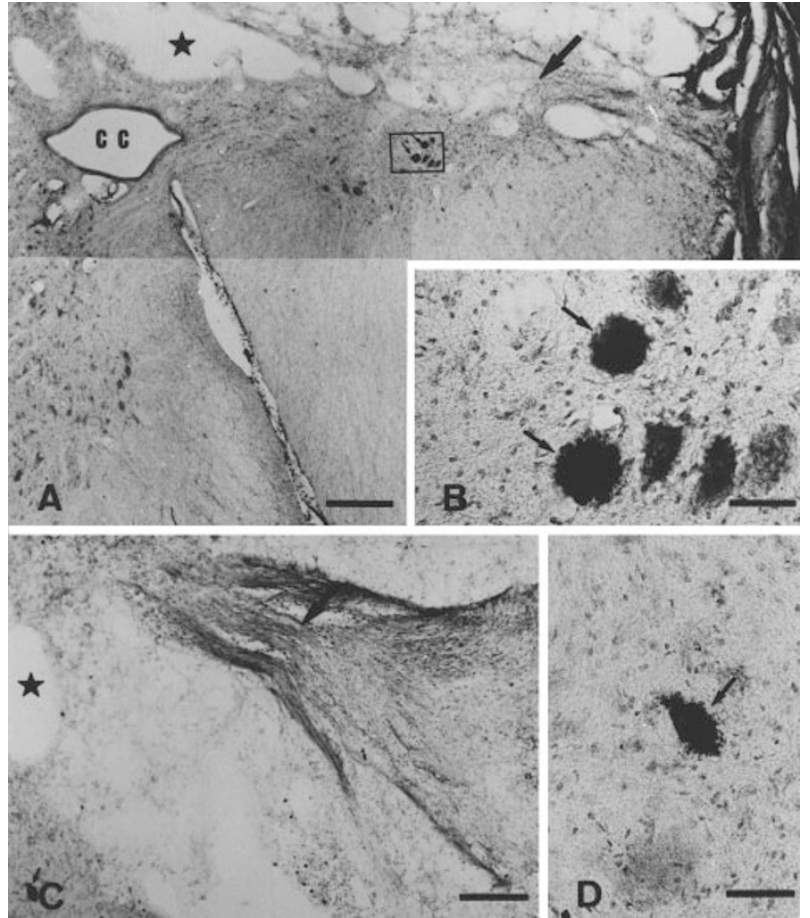

Figure 11 Cross section of the cervical spinal cord at the site of ventral rootlets reimplantation. (a) and (c) HRP labelled neurons (small arrows) assumed to be motoneurons, located in the ventral horn of the gray matter. Note the cystic cavities (star) around the reimplanted ventral rootlet (large arrow) and the swelling of the central canal (CC in a). (b and d) Higher magnification of labelled neurons (arrows) seen in a and d, respectively. Scale bars: $\mathrm{A}=400 \mu \mathrm{m} ; \mathrm{B}=50 \mu \mathrm{m}$; $\mathrm{C}=200 \mu \mathrm{m} ; \mathrm{D}=50 \mu \mathrm{m}$

C6
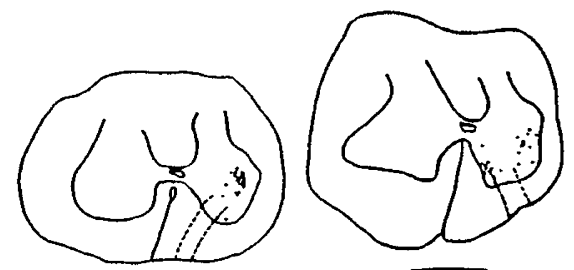

C7
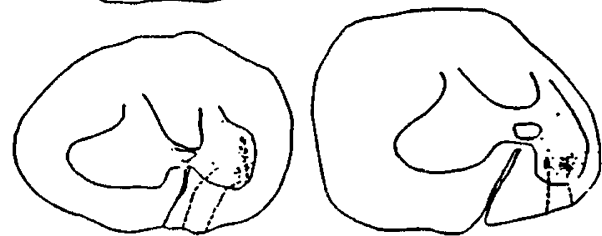

C8

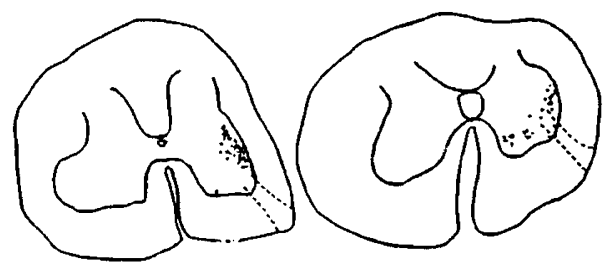

Figure 12 Camera Lucida drawings of the labelling pattern of the cervical spinal cord segments 6,7 and 8 , at the site of root reimplantation, 6 months p.o., in two dogs neurons through the site of injury. In experimental studies, in rats, ${ }^{5}$ cats $^{7}$ and monkeys, ${ }^{9}$ motoneurons of the cervical spinal cord have been shown to reinnervate the corresponding reimplanted ventral root. As expected, the present axonal tracing studies in the dog also demonstrated the reestablishment of axonal links between the ventral horn neurons, the reimplanted ventral roots and the corresponding motor nerves. In big mammals and in man, the long distance between the cervical spinal cord and the muscular effectors might be a limiting factor of anatomical and functional restoration that cannot obviously be considered in small animals such as rats, cats or even small monkeys (marmosets for instance). However, in medium and giant dog breeds, it is possible to evaluate the ability of axons to extend over distances exceeding $25 \mathrm{~cm}$, which is the distance separating the cervical spinal cord from the forearm, it should also be noted that in our previous study in medium size $\operatorname{dogs}^{12}$ we demonstrated that lumbar motoneurons could grow $15 \mathrm{~cm}$-axons into a blindended peripheral nerve graft. Taking these data into account, it can be inferred that the dog represents a worthwhile model for carrying out restorative strategies for both accidental and experimental nerve root avulsion.

\section{Advantages of the lateral surgical approach}

Compared to other approaches, our lateral surgical approach provides the following advantages: (1) It is easy to perform without major bleeding, provided that the interspinous branches of the internal vertebral venous plexus are ligated. Under these conditions, our nine dogs were kept alive during the six-month followup; (2) It allows a good visualization of up to three ventral roots $(\mathrm{C} 6, \mathrm{C} 7$ and $\mathrm{C} 8)$ that can be reimplanted in experimental or clinical conditions. After traumatic root avulsion, the ventral branches of the cervical nerves retract under the scalenius muscles. Consequently, it appears to be awkward to find them again and position their proximal tips into the vertebral canal via a ventral or a dorsal approach while this surgical step is easier using our lateral approach.

The postoperative neurological deficits consistently observed in the hindlimbs (paraparesia) and sympathetic ocular system (Claude Bernard-Horner's syndrome) appear to be the consequence of the development of intraspinal cysts at the lesion and grafting site (severing the tracts) rather than caused by the surgical approach itself. These lesions could result the avulsion procedure and/or from the reimplantation manoeuvre. Avulsion tears the rootlets away from the spinal cord and disrupts the radicular artery at the same time leading to a partial spinal cord ischemia. In the dog, $67 \%$ of the spinal cord segmental irrigation disappear after selective occlusion of the segmental arteries. ${ }^{18}$ Secondary bleeding due to deep reimplantation may also cause spinal cord damage. Bleeding as well as ischemia leads to spinal cord cavitation. As 
reimplantation was carried out immediately after avulsion, it is likely that both mechanisms contribute to the spinal cord damage we have observed.

Rotation of the spinal cord can also cause spinal injury but in this case, the resulting lesion is generally observed bilaterally. The spinal cord of the dog can be mobilized within physiological limits without any consequence on its irrigation. ${ }^{19}$ Our $15-20^{\circ}$ of rotation procedure was within these limits.

Axonal regrowth from neurons injured in the spinal cord The present experimentation evidences the capacity of dog spinal neurons to form and grow axonal extensions into ventral rootlets that have been deeply implanted into the spinal tissue. The grafted rootlets act by providing an environment which appears to be much more permissive to axonal growth and elongation than the surrounding injured CNS tissue of the adult animal. These findings are in full agreement with previous experiments where peripheral nerve grafts (PNG) or roots were implanted into the spinal cord in adult rodents, ${ }^{3,20}$ cats, ${ }^{8}$ monkeys ${ }^{9}$ or dogs. ${ }^{12}$ In PNG implantation studies in the dog, the average number of retrogradely HRP labelled cells was about 30 . This low value has been accounted for on the basis of technical considerations, since the number of labelled neurons was apparently correlated with the improvement of the surgical procedure with time. We think that higher is the number of rootlets reimplanted, higher is the number of labelled neurones (eg) larger is the surface of contact between CNS and peripheral nervous system elements). In contrast, cyst formation is a complication of neurosurgical trauma that disrupt the regeneration of spinal neurons. In fact, the low number of labelled neurons was directly correlated with the size of the cysts.

\section{Conclusion}

In this study, the surgical feasibility of ventral root reimplantation after their avulsion in the dog via a lateral approach is demonstrated. Injured canine spinal neurons extend axons through the reimplanted roots into the peripheral nerves. The reestablishment of functional motor units has now to be demonstrated by electrophysiological testing. The consequences of the surgical procedure on gait depends upon the surgical trauma at the time of reimplantation. We conclude that our lateral approach of the brachial plexus in the dog provides a good model to study the effects of surgical therapy after brachial plexus avulsion.

\section{Acknowledgements}

The study was supported by a grant from DGER 'Ministère de l'Agriculture', France and from the Institut pour la Recherche sur la Moelle Epinière (IRME). We are grateful to Upjohn Company for providing Solumedrol and Medrol. We thank Mr A Perignon for his drawing assistance, Mrs L Klukline for skilful technical assistance and Mrs G Butler-Browne for her critical reading of the manuscript.

\section{References}

1 Aguayo AJ. Axonal regeneration from injured neurons in the adult central nervous system. In: Cotman CW (ed). Synaptic plasticity. Guildford Press: New York, 1985; pp 457-484.

2 Vidal-Sanz M et al. Villegas-Perez MP, Thanos S, Aguayo AJ. Axonal regeneration and synapse formation in the superior colliculus by retinal ganglion cells in the adult rat. $J$ Neurosci 1987; 7: $2894-2909$.

3 Horvat J-C, Pecot-Dechavassine M, Mira J-C and Davarpanah Y. Formation of functional endplates by spinal axons regenerating through a peripheral nerve graft. A study in the adult rat. Brain Res Bull 1989; 22: $103-114$.

4 Jamieson AM and Eames RA. Reimplantation of avulsed brachial plexus roots: an experimental study in dogs. Int $J$ Microsurg 1980; 2: $75-80$.

5 Smith KJ and Kodama RT. Reinnervation of denervated skeletal muscle by central neurons regenerating via ventral roots implanted into the spinal cord. Brain Res 1991; 551: 221 - 229.

6 Hems TEJ, Clutton RE, Glasby MA. Repair of avulsed cervical nerve roots - an experimental study in sheep. J Bone Joint Surg (Br) 1994; 76-B: $818-823$.

7 Carlstedt $\mathrm{T}$ et al. Regeneration after spinal nerve root injury. Restor Neurol Neurosci 1990; 1: 289-295.

8 Hoffmann CFE, Thomeer RTWM and Marani E. Reimplantation of ventral rootlets into cervical spinal cord after their avulsion: an anterior surgical approach. Clin Neurol and Neurosurg 1993; 95: 112 - 118.

9 Carlstedt T, Hallin RG, Hedström KG and Nilsson-Remahl IAM. Functional recovery in primates after spinal cord implantation of avulsed ventral roots. $J$ Neurol Neurosurg Psychiatry 1993; 56: 649-654.

10 Carlstedt T. Functional recovery after ventral root avulsion and reimplantation in the spinal cord. Clinical Neurol Neurosurg 1993; 95: $109-111$.

11 Griffiths IR. Avulsion of the brachial plexus in the dog. In: Kirk RW (ed), Current Veterinary Therapy $6^{\circ}$. WB Saunders Co Philadelphia, 1977; pp 828-831.

12 Moissonnier P, Reviron T, Ye JH and Horvat J-C. Motoneurons of the injured spinal cord of the adult dog can grow lengthy axons into an autologous peripheral nerve graft. A retrograde axonal tracing study. Spinal Cord 1996; 34: 320-325.

13 Allam MWD, Lee DG, Nulsen FE and Fortune EA. The anatomy of the brachial plexus in the dog. Anat Rec 1952; 114: $173-180$.

14 Kitchell RL and Evans EE. The spinal nerves. In: Evans EE (ed), Miller's Anatomy of the $\operatorname{dog} 3^{\circ}$. WB Saunders Co, Philadelphia, 1993; pp 829-893.

15 Hohn RB, Rosen H, Bohning RH and Brown SG. Surgical stabilization of current shoulder luxation. Vet Clin North Am 1971; 1: $537-548$.

16 Mesulam MM. Tetramethylbenzidine for horseradish peroxidase histochemistry: a non-carcinogenic blue reaction product with superior sensitivity for visualizing neuronal afferents and efferents. J Histochem Cytochem 1978; 26: 106-117.

17 Wheeler SJ, Clayton-Jones DG and Wright JA. The diagnosis of brachial plexus disorders in dogs: a review of twenty-two cases. $J$ Small Anim Pract 1986; 27: 147 - 157.

18 Naito M, Owen JH and Bridwell KH. Blood flow direction in the lumbar nerve root. Spine, 1990, 15: 966-968.

19 Owen JH, Naito M and Bridwell KH. Relationship among level of distraction, evoked potential and spinal cord ischemia and integrity. Spine, 1990; 15: $852-857$.

20 Richardson P, Issa VMK, Aguayo AJ. Regeneration of long spinal axons in the rat. $J$ Neurocytol 1984; 13: $165-182$. 